\title{
Recurrence of Cholesteatoma - A Retrospective Study Including 1,006 Patients for More than 33 Years
}

\author{
Patrick Rønde Møller ${ }^{10}$ \\ Christina Nygaard Pedersen ${ }^{3}$ \\ Bjarki D. Djurhuus ${ }^{1,2}$ \\ ${ }^{1}$ Department of Ear, Nose and Throat Head and Neck Surgery, Zealand \\ University Hospital, Køge, Denmark \\ 2 Epidemiology, Biostatistics and Biodemography, Institute of Public \\ Health, University of Southern Denmark, Odense, Denmark \\ ${ }^{3}$ Department of Ear, Nose and Throat Head and Neck Surgery, Odense \\ University Hospital, Odense, Denmark
}

Address for correspondence Patrick Møller, Department of Ear, Nose and Throat Head and Neck Surgery, Zealand University Hospital, Lykkebækvej 1, Køge, 4600, Denmark (e-mail: drprmoeller@gmail.com).

Int Arch Otorhinolaryngol 2020;24:e18-e23.

\begin{abstract}
Introduction Cholesteatomas are benign tumors consisting of skin, and growing inside a retraction pocket in the tympanic membrane. Cholesteatomas can occupy the entirety of the middle ear, and are known for their osteolytic capabilities. Surgery is the only curative treatment for cholesteatomas.

Objective To describe the risk of recurrence after first-time surgically-treated middleear cholesteatoma (STMEC1) on the island of Funen from 1983 to 2015.

Methods Cases of STMEC1 were identified in the Danish National Hospital Register. The medical records were reviewed. Time-to-event analyses were applied. The ears were followed from STMEC1 to a secondary cholesteatoma, emigration, death, or end of follow-up.

Results Records from 1,006 patients with STMEC1 were reviewed. A total of 54 patients were submitted to surgery on both ears. The total sample consisted of 1,060 ears with STMEC $1 ; 300$ were children's ( $<16$ years) ears, and 760 were adult's ears. The total observation time was of 12,049 years.

The overall estimated proportion with recurrence 5 years after surgery was of $37 \%$ in children and of $15 \%$ in adults. The older the child was at the first surgery, the risk decreased by $7 \%$ peryear. In children, canal wall up (CWU) mastoidectomy without obliteration was associated with a hazard ratio for recurrence of 1.9 (95\% confidence

Keywords

- cholesteatoma

- recidivism

- recurrence

- surgery

- middle ear interval [95\% Cl]: 1.2-3.0) compared with CWU with obliteration.

Conclusion Compared with adults, children were had 2.6 times more risk of recurrence. Procedures performed without mastoidectomy had the lowest risk of recurrence. In children, obliteration was associated with a significantly lower risk of recurrence. However, patients were not randomized regarding the surgical approach; thus, the association between approach and risk of recurrence was likely influenced by confounding factors.
\end{abstract}

\section{Introduction}

Cholesteatoma is a condition that must be treated with surgery. A postauricular approach and mastoidectomy are often required. The two main types of mastoidectomy are "canal wall down" (CWD) and "canal wall up" (CWU). In CWD mastoidectomies, the posterior wall is removed, improving visualization but also resulting in an open cavity. In the CWU received

February 11, 2019

accepted

July 27, 2019
DOI https://doi.org/

10.1055/s-0039-1697989.

ISSN 1809-9777.
Copyright $\odot 2020$ by Thieme Revinter

Publicações Ltda, Rio de Janeiro, Brazil
License terms

()(1) $\Theta \circledast$ 
procedure, the posterior wall is preserved, maintaining the normal anatomy of the ear canal. ${ }^{1}$ A third procedure may involve obliteration of the mastoid cavity by applying materials that effectively obliterate the cavity. A recent review by van der Toom et $\mathrm{al}^{2}$ has shown recurrence rates as low as $4.6 \%$ for both CWD and CWU with obliteration. Other studies suggest recurrence rates of 5 to $17 \%$ for CWD without obliteration, and as high as 9 to $70 \%$ for CWU without obliteration, $^{3}$ thus suggesting the use of obliteration in future surgeries. However, the published literature on recurrence is often based on studies with surgeries performed by some of the world's most experienced ear surgeons; consequently, these procedures have low recurrence rates, and the authors advocate a specific surgical approach. There may be an issue of publication bias. Moreover, when doing epidemiologic research on cholesteatoma, large cohorts and long follow-up times are needed.

In Denmark, the opportunity for epidemiologic studies is unique due to a long tradition of collection of data on all inhabitants, ${ }^{4}$ and the unique civil registration number assigned to all persons living in Denmark since $1968 . .^{5}$ On the island of Funen, old medical records have been preserved, and they provide a unique opportunity to study the recurrence of cholesteatoma in a well-defined cohort with a long follow-up time.

The aim of the present study was to investigate the risk of recurrence after first-time surgically-treated middle-ear cholesteatoma (STMEC1) in a large, well-defined and unselected cohort with long follow-up, taking the surgical approach into consideration.

\section{Materials and Methods}

The Danish National Patient Register (NPR) was used for the identification of all patients with cholesteatoma surgery performed on the island of Funen in the period between January 1, 1983, and December 31, 2015.

All medical records from the identified patients were examined. For STMEC1, the date of hospitalization, the side of the ear, the surgical approach, any erosion of the posterior wall, any ossiculoplasty, and the date of recurrence were recorded.

Recurrence of cholesteatoma was defined as cholesteatoma in a subsequent surgery on the same ear. It proved impossible, by retrospective surgical chart review, to differentiate between a new cholesteatoma and a residual cholesteatoma, as well as to classify the cholesteatomas as flaccida or tensa cholesteatomas.

Patients without permanent residence in Denmark were excluded. Cases of congenital cholesteatomas and ear canal cholesteatomas were also excluded from the study.

Data on possible death or emigration to a foreign country were retrieved from the Patient Administrative System.

Possible surgery for recurrence elsewhere in Denmark was identified through the NPR, and, whenever possible, the relevant surgical charts were retrieved and included.

The surgical approaches were classified into 4 groups: ${ }^{1}$ no mastoidectomy; ${ }^{2}$ CWU without obliteration (CWU-obl); ${ }^{3}$ CWU with obliteration $(\mathrm{CWU}+\mathrm{obl}) ;^{4}$ and CWD.

\section{Statistical Analyses}

The Kaplan-Meier method and Cox regression analyses were applied.

In the analysis of recurrence, a second surgery for cholesteatoma on the same ear was considered an event, and the time since the first cholesteatoma surgery, as the underlying time variable. The ears were followed from the date of the first cholesteatoma surgery until the date of the second surgery (on Funen or elsewhere in Denmark), and censored at death, emigration to a foreign country, or due to end of follow-up (December 31, 2015), whichever came first. The interaction terms were tested by including them in the model.

Tests for violation of proportional hazards were performed using Schoenfeld residuals and log-log plot of survival. All statistical analyses were performed using the Stata (StataCorp, College Station, TX, US) software, version 14.

The analysis of the risk of contralateral disease was performed in a similar manner, with surgery on the opposite ear considered an event, and the time since cholesteatoma surgery on the first ear, as the underlying time variable.

The present study was approved by the Danish Data Protection Agency and the Danish Health Authorities.

\section{Results}

A total of 1,159 individuals had at least 1 cholesteatoma surgery registered in the NPR in the period between January 1, 1983, and December 31, 2015. the medical records were unavailable for 45 of these patients (3.9\%). The medical records in the remaining 1,114 patients were reviewed, and 63 patients (5.7\%) were found to have had had surgery before 1983 or at a department outside Funen, and were thus excluded. Of the remaining 1,051 patients, 2 (0.2\%) were excluded as they did not have permanent residency in Denmark. Of the remaining 1,049 individuals, 34 (3.1\%) had no cholesteatoma by medical record review, and they were excluded. Of the remaining 1,015 individuals, 2 were (0.2\%) were excluded as it was impossible to determine the type of surgery based on the surgical chart.

Of the remaining 1,013 individuals with verified cholesteatoma, 55 (5.1\%) had had cholesteatoma surgery on both ears, and there was a total of 1,068 ears with cholesteatoma. In these 1,068 ears, $5(0.5 \%)$ of the cholesteatomas were found to be congenital, and $3(0.3 \%)$ were found to be in the external ear canal. After excluding these 8 ears, the sample was composed of 1,060 ears of 1,006 patients (with 54 individuals with cholesteatoma surgery on both ears).

Of the 1,006 patients included, $25(2,49 \%)$ had subsequent cholesteatoma surgery recorded on the NPR at a hospital outside of Funen. The medical records could be obtained for review in 18 cases, and recurrence was confirmed in 12 of them. In 7 patients, the medical records from outside Funen were unobtainable, and we assumed that the patients had undergone surgery for recurrence on the date registered on the NPR.

The median age at STMEC1 was 33 years, and the total observation time was of 12,049 years. 
Table 1 Number of included ears by time period and surgical approach

\begin{tabular}{|l|l|l|l|l|l|}
\hline Period & No mast. (\%) & CWU-obl (\%) & CWU +obl (\%) & CWD (\%) & Total \\
\hline Children's ears & & & & & \\
\hline $1983-1984$ & $3(11)$ & $3(11)$ & $18(67)$ & $3(11)$ & 27 \\
\hline $1985-1989$ & $13(30)$ & $5(11)$ & $22(50)$ & $4(9)$ & 44 \\
\hline $1990-1994$ & $22(50)$ & $7(16)$ & $15(34)$ & $0(0)$ & 44 \\
\hline $1995-1999$ & $28(52)$ & $8(15)$ & $13(24)$ & $5(9)$ & 54 \\
\hline $2000-2004$ & $14(37)$ & $9(24)$ & $10(26)$ & $5(13)$ & 38 \\
\hline $2005-2009$ & $16(38)$ & $13(31)$ & $10(24)$ & $3(7)$ & 42 \\
\hline $2010-2015$ & $18(35)$ & $11(22)$ & $19(37)$ & $3(6)$ & 51 \\
\hline Total, children & $114(38)$ & $56(19)$ & $107(36)$ & $23(8)$ & 300 \\
\hline Adult's ears & & & & & $3(4)$ \\
\hline $1983-1984$ & $20(27)$ & $8(11)$ & $42(58)$ & $10(8)$ & 73 \\
\hline $1985-1989$ & $41(32)$ & $22(17)$ & $56(43)$ & $1(1)$ & 154 \\
\hline $1990-1994$ & $53(34)$ & $17(11)$ & $83(54)$ & $5(6)$ & 87 \\
\hline $1995-1999$ & $31(36)$ & $11(13)$ & $40(46)$ & $5(6)$ & 90 \\
\hline $2000-2004$ & $35(39)$ & $14(16)$ & $36(40)$ & $5(5)$ & 99 \\
\hline $2005-2009$ & $44(44)$ & $30(30)$ & $20(20)$ & $10(8)$ & 128 \\
\hline $2010-2015$ & $46(36)$ & $18(14)$ & $54(42)$ & $39(5)$ & 760 \\
\hline Total, adults & $270(36)$ & $120(16)$ & $331(44)$ & $62(6)$ & 1060 \\
\hline Total & $384(36)$ & $176(17)$ & $438(41)$ & & \\
\hline
\end{tabular}

Abbreviations: CWD, canal wall down; CWU + obl, canal wall up with obliteration; CWU-obl, canal wall up without obliteration; No mast., No mastoidectomy.

Of the 1,060 ears, 300 (28\%) were children's ( $<16$ years) ears, and 760 (72\%) were adult's ears. The number of ears included by time period and surgical approach is summarized in - Table $\mathbf{1}$.

In children's ears 191 (64\%) of the cholesteatomas were in male patients, and in adult's ears, 441 (58\%) were in male patients.

Recurrence was found in 133 out of 300 children's ears (44\%), and in 162 of 760 (21\%) adult's ears.

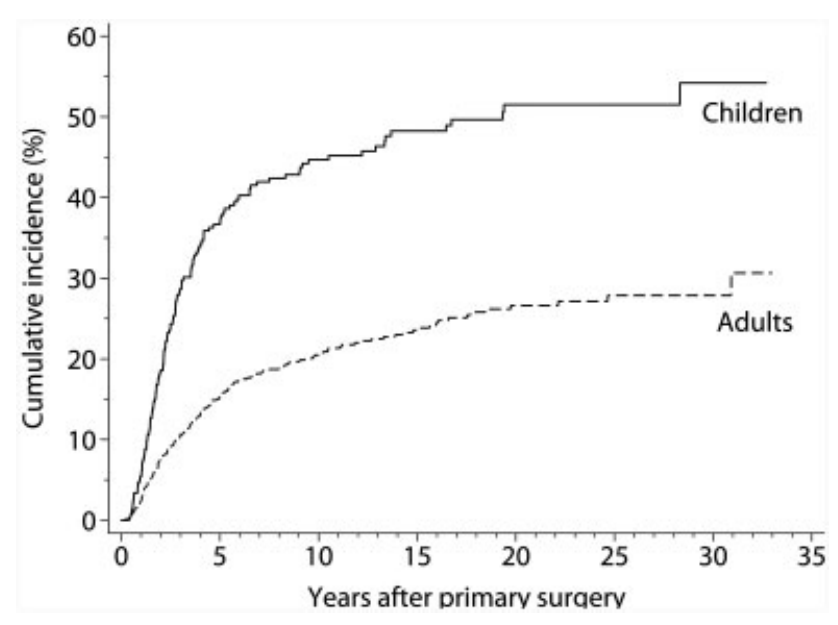

Fig. 1 Kaplan-Meier failure function illustrating the cumulative incidence proportions with recurrence of cholesteatoma in children (aged $<16$ years) and in adults.

\section{Time-to-Event Analyses}

-Fig. 1 demonstrates a much higher risk of recurrence in children compared with adults. After 5 years, an estimated 111 (37\%) (95\% confidence interval [95\%CI]: 31-43\%) of the children had had recurrence compared with 114 (15\%) (95\% CI: $13-18 \%)$ of adults, a rate that is 2.6 times higher.

\section{Children}

The rate of recurrence after 5 years (-Fig. 2) was lower in children without the need of mastoidectomy (23\%; $95 \% \mathrm{CI}$ : 16-32\%), and higher in children with CWU-obl (58\%; (95\%CI: $45-71 \%)$. The rate of recurrence in cases of CWU + obl was of $44 \%$ (95\%CI: 35-55\%) after 5 years.

The multivariate analysis ( $\mathbf{- T a b l e ~} \mathbf{2}$ ) revealed a statistically higher risk of recurrence associated with CWU-obl compared with CWU + obl (hazard ratio [HR]: 1.91; 95\%CI: 1.20-3.02).

The HR for age at first surgery was of 0.93 , that is, the older the child was at STEMC1, the risk of recurrence was reduced by $7 \%$ per year.

None of the defined time periods differed significantly from the baseline period. However, a clear trend of decrease in cholesteatoma recurrence with increasing time period was observed, and the test for trend revealed an HR of 0.96 (95\%CI: 0.94-0.98; $p<0.001$ ).

\section{Adults}

As in the group of children, in adults, the rate of recurrence was lower in the group with no mastoidectomy $(12 \% ; 95 \% \mathrm{CI}$ : 


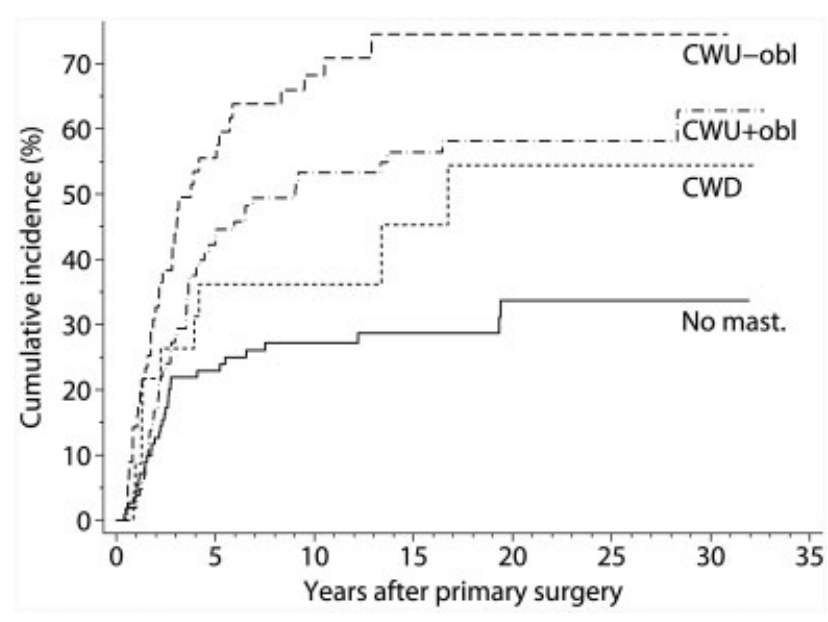

Fig. 2 Kaplan-Meier failure function illustrating the cumulative incidence proportions with recurrence of cholesteatoma in children (aged < 16 years) by surgical approach. Abbreviations: CWD, canal wall down; CWU + obl, canal wall up with obliteration; CWU-obl, canal wall up without obliteration; No mast.: no mastoidectomy.

Table 2 Results of the Cox regression model estimating the hazard ratio of developing recurrent cholesteatoma by covariates in children (aged $<16$ years)

\begin{tabular}{|c|l|l|l|}
\hline & HR & $95 \% \mathrm{Cl}$ & $p$-value \\
\hline Surgical approach & & & \\
\hline No mastoidectomy & 0.55 & $0.33-0.94$ & 0.027 \\
\hline CWU without obliteration & 1.91 & $1.20-3.02$ & 0.006 \\
\hline CWU with obliteration & Ref. & - & - \\
\hline CWD & 1.27 & $0.54-2.98$ & 0.219 \\
\hline Age at first surgery & 0.93 & $0.88-0.98$ & 0.010 \\
\hline Year of primary surgery & & & \\
\hline $1983-1984$ & 1.70 & $0.88-3.30$ & 0.114 \\
\hline $1985-1989$ & 1.54 & $0.87-2.73$ & 0.136 \\
\hline $1990-1994$ & 1.45 & $0.80-2.65$ & 0.220 \\
\hline $1995-1999$ & Ref & - & - \\
\hline $2000-2004$ & 0.75 & $0.38-1.48$ & 0.411 \\
\hline $2005-2009$ & 0.81 & $0.42-1.56$ & 0.533 \\
\hline $2010-2015$ & 0.66 & $0.28-1.34$ & 0.219 \\
\hline Sex & & & \\
\hline Female & Ref. & - & - \\
\hline Male & 0.75 & $0.52-1.09$ & 0.136 \\
\hline Ossiculoplasty & & & \\
\hline No & Ref. & - & - \\
\hline Yes & 1.39 & $0.82-2.34$ & 0.218 \\
\hline Erosion of posterior wall & & & \\
\hline No & 0.67 & $0.37-1.22$ & 0.190 \\
\hline Yes & & & - \\
\hline
\end{tabular}

Abbreviations: 95\% Cl, 95\% confidence interval; CWD, canal wall down; $\mathrm{CWU}$, canal wall up; HR, hazard ratio; No mast., no mastoidectomy; Ref., reference.

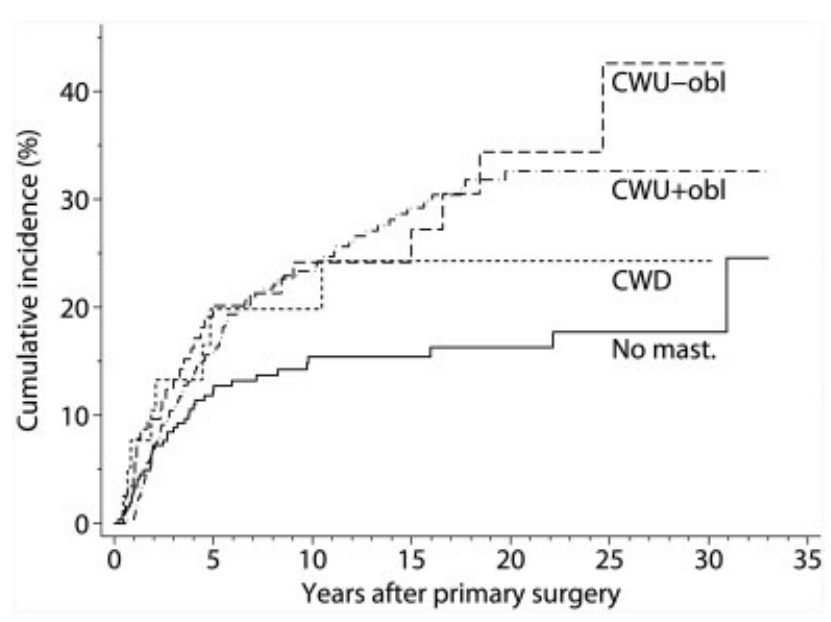

Fig. 3 Kaplan-Meier failure function illustrating the cumulative incidence proportions with recurrence of cholesteatoma in adults (aged $\geq 16$ years) by surgical approach. Abbreviations: CWD, canal wall down; CWU + obl, canal wall up with obliteration; CWU-obl, canal wall up without obliteration; No mast.: no mastoidectomy.

$8-16 \%)$ after 5 years, and higher in the group submitted to CWU-obl (20\%; 95\%CI: 14-29\%) (-Fig. 3).

In adults, there was no significant difference in CWU-obl compared with CWU + obl ( - Table 3$)$.

\section{Cholesteatoma on Contralateral Ears}

The risk of STMEC1 on the contralateral ear was higher in children ( - Fig. 4) (6\%; 95\%CI: 3-9\%) 5 years after STMEC1 on the first ear. This rate was of $8 \%$ (95\%CI: 5-12\%) 20 years after STMEC1 on the first ear. In adults, these numbers were $4 \%$ (95\%CI: 3-6\%) and 5\% (95\%CI: 4-8\%) respectively.

\section{Discussion}

Our study showed that the overall risk of recurrence of cholesteatoma 5 years after primary surgery was 2.6 times higher in children compared with adults (38\% versus $14 \%$ respectively). Moreover, the older the children were at the STMEC1, the risk decreased by $7 \%$ per year. This is in line with prior findings of high recurrence rates in children, ${ }^{6-8}$ and may come from a better Eustachian tube function during childhood $^{9,10}$ which lowers the risk of negative middle ear pressure and retraction pockets, thus lowering the risk of a new cholesteatoma.

No mastoidectomy was associated with a considerably lower risk of recurrence in both children and adults. This was most likely because of less advanced cases of the disease and possibly because of the type of cholesteatoma.

In children, when mastoidectomy was performed, CWUobl was associated with a statistically significant higher risk of recurrence (HR: 1.9) compared with $\mathrm{CWU}+$ obl. This supports the findings of other studies. ${ }^{11-15}$ However, the patients in the present study were not randomized; thus, this association may also be the result of confounding factors. Factors such as location and extent of the cholesteatoma, the skill of the surgeon, as well as signs of infection, may all have influenced both the risk of recurrence and the choice to 
Table 3 Results of the Cox regression model estimating the hazard ratio of developing recurrent cholesteatoma by covariates in adults (aged $\geq 16$ years)

\begin{tabular}{|c|l|l|l|}
\hline & HR & $95 \% C l$ & $p$-value \\
\hline Surgical approach & & & \\
\hline No mastoidectomy & 0.65 & $0.42-0.99$ & 0.047 \\
\hline CWU without obliteration & 1.19 & $0.76-1.86$ & 0.444 \\
\hline CWU with obliteration & Ref. & - & - \\
\hline CWD & 0.82 & $0.36-1.88$ & 0.643 \\
\hline Age at first surgery & 0.98 & $0.97-0.99$ & $<0.001$ \\
\hline Year of primary surgery & & & \\
\hline $1983-1984$ & 2.02 & $1.06-3.85$ & 0.033 \\
\hline $1985-1989$ & 0.91 & $0.47-1.77$ & 0.775 \\
\hline $1990-1994$ & 1.76 & $0.98-3.18$ & 0.060 \\
\hline $1995-1999$ & Ref. & - & - \\
\hline $2000-2004$ & 1.40 & $0.72-2.73$ & 0.322 \\
\hline $2005-2009$ & 1.18 & $0.57-2.43$ & 0.655 \\
\hline $2010-2015$ & 1.90 & $0.93-3.89$ & 0.080 \\
\hline Sex & & & \\
\hline Female & Ref, & - & - \\
\hline Male & 1.20 & $0.87-1.65$ & 0.279 \\
\hline Ossiculoplasty & & & \\
\hline No & Ref. & - & - \\
\hline Yes & 1.12 & $0.73-1.71$ & 0.618 \\
\hline Erosion of posterior wall & & & \\
\hline No & Ref. & - & - \\
\hline Yes & 1.21 & $0.85-1.89$ & 0.252 \\
\hline
\end{tabular}

Abbreviations: $95 \% \mathrm{Cl}$, 95\% confidence interval; CWD, canal wall down; CWU + obl, canal wall up; HR, hazard ratio; No mast., no mastoidectomy; Ref., reference.

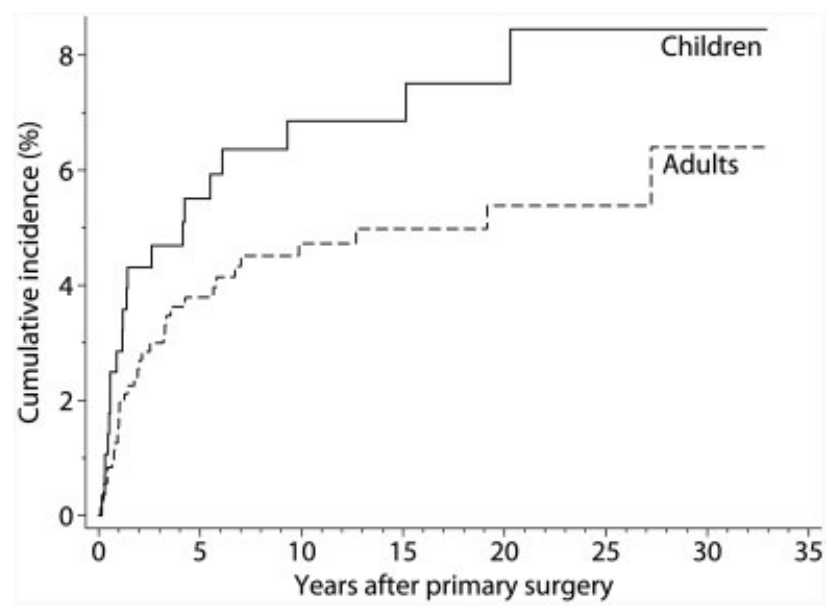

Fig. 4 Kaplan-Meier failure function illustrating the cumulative incidence proportions with cholesteatoma surgery performed on the contralateral ear in children (aged $<16$ years) and in adults. obliterate or not obliterate. Unfortunately, the data provided did not enable us to correct this. The material used for obliteration at that time on Funen was hydroxyapatite granule, and the obliteration was performed with the following technique: firstly, the mastoidectomy was performed using the canal wall up procedure, and the cholesteatoma was completely removed. Secondly, the mastoid and the middle ear were completely separated using cartilage and temporalis fascia, and the mastoid was tightly obliterated using hydroxy apatite granules, cortical bone chips and fibrin glue. Thirdly, ossicular reconstruction and myringoplasty with temporal fascia or cartilage were performed, thus restoring the ability to hear.

Most recurrences occurred within 5 years after the primary surgery. However, there still was a considerable risk of recurrence after 5 years, and it could be argued that a 10 -year follow-up period is appropriate in some cases.

The finding of cholesteatoma in the contralateral ear in $8 \%$ of the children and in $5 \%$ of the adults after 20 years of followup underscores the importance of checking the contralateral ear in individuals with cholesteatoma.

\section{Strengths and Limitations}

The present study has several strengths. Firstly, the study population encompassed a well-defined and unselected cohort. Secondly, the possibilities for the identification of patients and follow-up were exceptional due to the unique personal identification numbers, the preservation of medical records on Funen, the possibility to identify recurrence outside of Funen by the NPR, and the access to data on death and emigration to foreign countries in the patient administrative system. Thirdly, the analyses performed were appropriate time-to-event analyses. Lastly, the study population was large, and included both children and adults, as well as different surgical approaches, enabling direct comparisons.

The present study also has some limitations. Firstly, a major limitation was the lack of randomization combined with the lack of possibility to sufficiently adjust for parameters affecting the choice of surgical approach. An obvious result caused by confounding factors was the association between no mastoidectomy and a lower risk of recurrence. The underlying reason for the association between no obliteration and a lower risk of recurrence, which may also very well be a confounding factor, was more obscure. However, we considered unfeasible the possibility of conducting a study of this size, and especially with this follow-up time, as a prospective and randomized trial.

Secondly, there is the inherent risk of misclassification in the records. However, the medical records were reviewed, and all false positive cases in the NPR were excluded from the study. The completeness of the NPR has previously been estimated to be of $91 \%$ for STMEC $1 .{ }^{16}$ However, the number of cases missed due to this has previously been estimated to be limited. ${ }^{17}$ Thirdly, the risk of individuals only undergoing one cholesteatoma surgery not being registered in the NPR is greater than the risk for individuals undergoing several cholesteatoma surgeries. This may have slightly increased the estimated risk of recurrence. 
Lastly, it proved impossible to differentiate between a new cholesteatoma and a residual cholesteatoma, as well as to classify the cholesteatomas as flaccida or tensa cholesteatomas in the present retrospective study.

\section{Conclusion}

In the present study, which included 1,006 patients and a total observation time of 12,049 years, we found that, compared with adults, children had 2.6 times more risk of recurrence. The older the child was at the first surgery, the risk decreased by $7 \%$ per year.

Ears without the need of mastoidectomy had the lowest risk of recurrence. In children treated with CWU, obliteration was associated with a risk of recurrence that was significantly lower than that of no obliteration. However, the patients were not randomized with regards to surgical approach, and the demonstrated association between surgical approach and risk of recurrence was likely influenced by confounding factors.

The estimated rate of cholesteatoma surgery on the contralateral ear 5 years after cholesteatoma surgery on the first ear was of $6 \%$ in children and of $4 \%$ in adults.

The high risk of recurrence and a considerable risk of a subsequent cholesteatoma on the contralateral ear underscore the importance of follow-up after cholesteatoma surgery.

Source of Financial Support

The Hans Skouby Foundation.

Conflict of interest

The authors have none to disclose.

\section{References}

1 Sie KC. Cholesteatoma in children. Pediatr Clin North Am 1996;43 (06):1245-1252

2 van der Toom HFE, van der Schroeff MP, Pauw RJ. Single-Stage Mastoid Obliteration in Cholesteatoma Surgery and Recurrent and Residual Disease Rates: A Systematic Review. JAMA Otolaryngol Head Neck Surg 2018;144(05):440-446
3 Tomlin J, Chang D, McCutcheon B, Harris J. Surgical technique and recurrence in cholesteatoma: a meta-analysis. Audiol Neurotol 2013;18(03):135-142

4 Thygesen LC, Daasnes C, Thaulow I, Brønnum-Hansen H. Introduction to Danish (nationwide) registers on health and social issues: structure, access, legislation, and archiving. Scand J Public Health 2011;39(7, Suppl)12-16

5 Pedersen CB. The Danish Civil Registration System. Scand J Public Health 2011;39(7, Suppl)22-25

6 McGuire JK, Wasl H, Harris T, Copley GJ, Fagan JJ. Management of pediatric cholesteatoma based on presentations, complications, and outcomes. Int J Pediatr Otorhinolaryngol 2016;80:69-73

7 Sanna M, Zini C, Gamoletti R, et al. The surgical management of childhood cholesteatoma. J Laryngol Otol 1987;101(12):1221-1226

8 Darrouzet V, Duclos JY, Portmann D, Bebear JP. Preference for the closed technique in the management of cholesteatoma of the middle ear in children: a retrospective study of 215 consecutive patients treated over 10 years. Am J Otol 2000;21(04):474-481

9 Bylander A, Tjernström O. Changes in Eustachian tube function with age in children with normal ears. A longitudinal study. Acta Otolaryngol 1983;96(5-6):467-477

10 Sadler-Kimes D, Siegel MI, Todhunter JS. Age-related morphologic differences in the components of the eustachian tube/middle ear system. Ann Otol Rhinol Laryngol 1989;98(11):854-858

11 Gantz BJ, Wilkinson EP, Hansen MR. Canal wall reconstruction tympanomastoidectomy with mastoid obliteration. Laryngoscope 2005;115(10):1734-1740

12 Mercke U. The cholesteatomatous ear one year after surgery with obliteration technique. Am J Otol 1987;8(06):534-536

13 van Dinther JJS, Vercruysse J-P, Camp S, et al. The Bony Obliteration Tympanoplasty in Pediatric Cholesteatoma: Long-term Safety and Hygienic Results. Otol Neurotol 2015;36(09):1504-1509

14 Vercruysse J-P, De Foer B, Somers T, Casselman JW, Offeciers E. Mastoid and epitympanic bony obliteration in pediatric cholesteatoma. Otol Neurotol 2008;29(07):953-960

15 Lee WS, Choi JY, Song MH, Son EJ, Jung SH, Kim SH. Mastoid and epitympanic obliteration in canal wall up mastoidectomy for prevention of retraction pocket. Otol Neurotol 2005;26(06): $1107-1111$

16 Djurhuus BD, Skytthe A, Faber CE. Validation of the cholesteatoma diagnosis in the Danish National Hospital Register. Dan Med Bull 2010;57(10):A4159

17 Djurhuus BD, Skytthe A, Christensen K, Faber CE. Cholesteatoma in Danish children - a national study of changes in the incidence rate over 34 years. Int J Pediatr Otorhinolaryngol 2015;79(02):127-130 